Economía, Sociedad y Territorio, vol. vIII, núm. 26, 2008, 451-476

\title{
Evaluación rural participativa: uso de los recursos naturales en la reserva de la biosfera El Vizcaíno, BCS, México
}

\author{
Magdalena Lagunas-VázqueZ* \\ Luis Felipe BeltráN-Morales*** \\ José Urciaga-García* \\ Alfredo Ortega-Rubio**
}

\begin{abstract}
In this paper we describe our experience during a participative research where we emphasise the importance of linking the different social players (in this case, protected rural areas), the academic sector and non-governmental organisations. We analyse the results of a participative rural appraisal (PRA or ERP in Spanish) carried out in El Vizcaino biosphere reserve, Baja California Sur, Mexico. Apart from identifying the problems perceived by the social players and their proposals to solve them, we obtained information about the usage of natural resources by the residents of the different communities and formulated a joint plan for their exploitation.
\end{abstract}

Keywords: participative rural appraisal, protected natural areas, natural resource usage, rural communities.

\section{Resumen}

En este artículo se describe la experiencia de investigación participativa donde se destaca la importancia de la vinculación entre los actores sociales (en este caso de áreas rurales protegidas), el sector académico y las organizaciones no gubernamentales. Se analizan los resultados de una evaluación rural participativa (ERP) llevada a cabo en la Reserva de la Biosfera El Vizcaíno, BCS, México. Además de identificar lo que los actores sociales perciben como problemas y las formas en que proponen vías de solución, se logró obtener información acerca del uso de los recursos naturales por parte de los residentes de las comunidades y se formuló un plan comunitario de uso de los mismos.

Palabras clave: evaluación rural participativa (ERP), áreas naturales protegidas, uso de recursos naturales, comunidades rurales.

* Universidad Autónoma de Baja California Sur. Correos-e: mlagunas@uabcs.mx, jurciaga@uabcs.mx.

** Centro de Investigaciones Biológicas del Noroeste s.c. (CIBNOR). Correos-e: aortega@cibnor.mx, lbeltran04@cibnor.mx. 


\section{Introducción}

Durante las últimas décadas, en América Latina se ha dado una profunda crisis económica y política, con procesos de ajuste estructural y tendencias de desarrollo de corte neoliberal; con escasa participación social en la toma de decisiones sobre estos cambios, antes, más bien, autoritarios y escasamente transparentes; debido, sobre todo, a sistemas políticos muy centralizados, elitistas, antidemocráticos y corruptos. Como resultado de esto, en la región se acentuó la pobreza, se incrementó la concentración de riqueza y se multiplicaron los problemas sociales (violencia, delincuencia, violación a los derechos humanos, discriminación y narcotráfico) (Valarezo, 1995).

Organismos de Naciones Unidas, ONU (como la organización para la Agricultura y la Alimentación, FAO, y el Programa para el Medio Ambiente, PNUMA), señalan firmemente la necesidad de nuevas perspectivas y maneras de promover el desarrollo rural para detener o revertir el deterioro ambiental, donde se incluyan sobre todo criterios socialmente más participativos. Durante décadas, el gobierno mexicano mantuvo en el campo una política de buenas intenciones y manejos paternalistas (Martínez et al., 1999). Es decir, los programas y apoyos de desarrollo rural no trataban de crear alternativas viables para los campesinos, más bien, éstos se basaban en elevar la producción a bajos costos para subsidiar el crecimiento industrial y, por ende, el crecimiento urbano, así como mantener la paz en el campo con actitudes paternalistas y control político. La respuesta campesina fue justamente la indiferencia y la aceptación de planes y proyectos que no representaban sus intereses, mientras que la realidad se agravaba ya que muchas veces las actividades y tecnologías aplicadas no eran las adecuadas para las características ambientales de la zona (Martínez et al., 1999).

Considerando que en México gran parte de las áreas rurales se componen principalmente de identidades colectivas que se conforman de propiedades comunales (forma de organización tradicional de los pueblos indios mexicanos), en la actualidad se reconocen como terrenos ejidales o ejidos (González, 2001). Generalmente, las comunidades rurales, debido a las condiciones de pobreza en que se encuentran (ocho de cada diez familias rurales son pobres y cuatro de esas ocho están en extrema pobreza), se ven obligados a realizar una explotación intensiva de sus recursos y a utilizar en determinadas actividades predios que 
no tienen el potencial adecuado (Carabias et al., 1994). Por ello, consideramos que las comunidades rurales dependen en gran medida de sus recursos naturales, lo que demuestra su importancia para resguardar un área natural, ya que ellas pueden operar como aliadas de la protección biológica y la diversidad genética in situ (Bocco et al., 2000).

Actualmente, la preocupación de diferentes sectores sociales ante la crisis ambiental por la que atraviesa la humanidad hace necesaria la búsqueda de nuevas alternativas que favorezcan la protección, conservación y uso adecuado de los recursos naturales, en especial en regiones bajo fuertes presiones ambientales y socioeconómicas (Bocco et al., 2000).

La experiencia que se describe en este trabajo aborda el papel de la investigación participativa entre áreas rurales inmersas en un área natural protegida (ANP), el sector académico y organizaciones no gubernamentales (ONG). El objetivo principal de este estudio es facilitar a la comunidad de Bonfil una evaluación del uso de sus recursos naturales, priorizar los problemas y soluciones en el manejo de tales recursos (por medio de la ERP) y ofrecer pautas para formular un plan de manejo comunitario de los recursos naturales. La hipótesis planteada por el grupo investigador fue: la comunidad de Bonfil hace uso de los recursos naturales cercanos a sus casas y su cultura y costumbres están influenciadas por las características ambientales de su entorno natural.

\section{Evaluación rural participativa}

La evaluación rural participativa (ERP) estima que la colaboración social es un ingrediente fundamental para elaborar proyectos, impulsar un mejoramiento sustantivo en la calidad de vida local y conservar los recursos naturales. El método de ERP derivó de la evaluación rural rápida (ERR) y se utilizó por primera vez a comienzos de la década de los ochenta (Chambers, 1983, 1991, 1993). Constituye un enfoque participativo o un marco metodológico para obtener, evaluar y analizar, en un periodo corto, información relevante sobre las realidades rural y urbana. Su propósito es dar prioridad a los problemas esenciales, identificar y planificar posibles acciones concretas y estimular la discusión (Chambers, 1992a, 1992b). Este enfoque involucra a la población local en la investigación y análisis, considera el conocimiento de la población local como punto de partida y pone énfasis en 
el aprendizaje mutuo entre la población. De acuerdo con Geertz (1994), cada grupo social tiene una manera de interpretar la realidad y relacionarse con ella; desde esta dimensión (subjetiva de la vida social) dan sentido y orientación a sus acciones, por lo que se considera imprescindible ubicar y reconocer el conocimiento local.

Las herramientas participativas y el trabajo comunitario son procesos que permiten crear espacios para compartir el conocimiento local (Centro Internacional para el Ambiente y el Desarrollo, 1993; Chambers, 1996), las experiencias cotidianas y el conocimiento ecológico tradicional de las comunidades, lo cual posibilita la libre expresión, el análisis y la discusión de la propia realidad local (Vázquez y Montenegro, 1999; Berkes et al., 2001).

La ERP se basa en la premisa de que es frecuente que la solución a los problemas sobre el manejo de recursos se encuentre en la propia comunidad, por lo que definir tales problemas requiere del consenso entre los diferentes grupos de usuarios, tanto para priorizar las dificultades como para proponer posibles soluciones; es decir, la evaluación-planeación en conjunto sobre el uso de los recursos naturales en el ámbito local (Pretty et al., 1991, 1993, 1995). Así, la ERP sólo podrá ser útil cuando se den estas condiciones fundamentales para la negociación, donde las comunidades están convencidas de participar (Leewis, 2000).

El enfoque de la ERP incluye los términos rural y evaluación, pero Robert Chambers, uno de los principales representantes en este campo, observa que los términos a veces son un problema, pues rural es un término al que se le dan muchas aplicaciones, y la palabra evaluación es muy limitada porque la ERP es un proceso que implica mucho más que la simple evaluación (Chambers, 1995). Para otros autores, evaluar es una actividad participativa que requiere una perspectiva y un equipo interdisciplinarios, tanto evaluadores internos (representantes de la comunidad) como externos (técnicos, facilitadores), y tiene un proceso cíclico cuyo objetivo principal es fortalecer el sistema que se evalúa así como la metodología utilizada (Macías et al., 2006).

La ERP se ha usando ampliamente en África y en Asia, aunque es menos conocida en América Latina. Hay quienes consideran que aunque el lenguaje de este método no es tan explícito como los enfoques auténticamente latinoamericanos (por ejemplo, la investigación-acción-participativa, IAP), comparte algunos de sus elementos clave, al tiempo que ofrece una amplia variedad de 
métodos y técnicas avalados por la experiencia. Dentro de los elementos esenciales de la ERP se encuentran (Chambers, 1995):

- Equipo multidisciplinario con una variedad de habilidades y antecedentes.

- Claridad en cuanto al espacio, proyecto, personas, géneros y costumbres.

- Uso de diferentes métodos, fuentes, disciplinas e informantes en diferentes lugares para realizar controles cruzados en aproximaciones sucesivas.

- Los miembros de la comunidad participan en la evaluación.

- Los investigadores principales trabajan en contacto directo, cara a cara y en el campo.

- Cambio de papeles: se aprende de y con la comunidad rural acerca de sus criterios y categorías.

- Ignorancia óptima e imprecisión aproximada: al no descubrir ni medir más de lo necesario y pasar por alto lo que no es necesario.

- Análisis en el lugar mismo para determinar qué se debe sugerir.

- Preferencia por la conducta, las actitudes y la afinidad por encima de los métodos.

- Cultura para compartir la información, los métodos, el alimento, las experiencias de campo, etcétera.

- Conciencia autocrítica en relación con nuestras actitudes y conductas.

El método de ERP se ha aplicado en comunidades de diversas regiones de África, Asia, Europa y Centroamérica (Centro Internacional para el Ambiente y el Desarrollo, 1993). En México se tienen experiencias con enfoque participativo desde 1965 (corrientes filosóficas influyentes: teología de la liberación de Boff, educación popular de Freire, investigación participativa militante de Fals Borda y Rahman y democracia participativa y ecología social de Murray Bookchin), y a finales de 1980 la versión inglesa del diagnóstico rural rápido (Macías et al., 2006).

En los últimos 10 años, en México aumentó el uso de alternativas para el desarrollo participativo, tanto enfoques y métodos de participación social como diagnósticos y evaluaciones en los ámbitos comunitario y regional, entre los que destacan: la evaluación rural participativa, el diagnóstico rural rápido, la investi- 
gación participativa y la investigación-acción-participativa (IAP), con lo que la colaboración en la planeación del desarrollo ha sido ampliamente aceptada como herramienta metodológica en programas del sector rural de los dos últimos gobiernos. Por lo que tanto los gobiernos como algunas instituciones internacionales de financiamiento (como el Banco Mundial) han impulsado la utilización de estas metodologías (Negrete y Bocco, 2003; Macías et al., 2006).

De esta manera, en México se tienen experiencias recientes de uso de metodologías para la participación social en las que se aplicó una herramienta o la combinación de varias, como en el caso del diseño e implementación del sistema de monitoreo y evaluación participativa: la experiencia en Calakmul, Campeche, donde, además de la aplicación de la ERP, se utilizaron diversas metodologías de la IAP; el ordenamiento territorial en Santa Martha, municipio de Chenalhó, Chiapas (Pool et al., 1997); el ordenamiento ecológico y actividades de ecoturismo y educación ambiental en la comunidad de Nuevo San Juan Parangaricutiro, Michoacán (Bocco et al., 2000). El Grupo de Estudios Ambientales, A.C. tiene más de 10 años de experiencia de interacción entre 30 comunidades marginadas y una organización campesina regional en varias líneas de trabajo, entre las que destacan: el Programa de Manejo Campesino de Recursos Naturales y Sistemas Agroalimentarios que desarrollan conjuntamente las comunidades, la Sociedad de Solidaridad Social (sss) Sanzekan Tienmi; y el Proyecto de Conservación de la Biodiversidad por Comunidades Indígenas de los estados de Oaxaca, Michoacán y Guerrero (Coinbio), que desde hace tres años incorpora recursos financieros nacionales e internacionales para apoyar algunas iniciativas de ejidos y comunidades en materia de fortalecimiento de capacidades locales para un mejor conocimiento de su biodiversidad, mediante, entre otras actividades, talleres participativos comunitarios e inventarios florísticos. El Coinbio es un proyecto que opera en los tres estados mencionados a partir del año 2001 y su principal objetivo es apoyar y fortalecer las iniciativas comunitarias de conservación de ecosistemas y biodiversidad a partir de los propios esquemas y modelos comunitarios (Anta, 2006; Granich, 2006). 


\section{Área de estudio}

En Baja California Sur la mayoría de las localidades son rurales: de un total de 2,743 poblados, $99.3 \%$ son rurales, sin embargo, la mayoría de las personas habita en las pocas localidades urbanas; ya que de 424,041 habitantes con que cuenta el estado, sólo $18.7 \%$ vive en áreas rurales (INEGI, 2000a). Prácticamente toda la entidad está dividida en terrenos ejidales, en total son 100 ejidos (INEGI, 2000b). De acuerdo con las costumbres y la cultura de la población rural, es importante reconocer y caracterizar a estas comunidades, sobre todo en cómo usan y manejan sus recursos naturales.

De la superficie total de BCS, $40 \%$ cuenta con algún decreto que la establece como área natural protegida (ANP), con lo que se ubica como el primer estado que aporta mayor cantidad de territorio con ANP al país (CONANP, 1999). Esto nos indica su gran importancia ecológica nacional e internacional por las características de los ecosistemas que lo conforman: elevada biodiversidad, endemismo, presencia de especies en peligro de extinción, así como el potencial económico de los recursos naturales del territorio. Más adelante se describe el aspecto biofísico de esta región.

En el conjunto de ANP del estado se encuentra la reserva de la biosfera El Vizcaíno, la segunda más extensa de América Latina con 2'546,790 ha. Dentro de ésta se ubica el ejido Bonfil -área de estudio del presente trabajo-, que es un conjunto de comunidades rurales que pertenecen al municipio de Mulegé, al norte de Baja California Sur. El ejido Bonfil obtuvo resolución presidencial, en el Diario Oficial de la federación el 27 de junio de 1975 , para dotarlo con 514,575 ha de territorio y así convertirlo en uno de los ejidos de mayor extensión territorial del país. Aproximadamente 50\% del territorio ejidal está dentro de la reserva de la biosfera El Vizcaíno.

Para el desarrollo del Taller de evaluación rural participativa (TERP) se regionalizó el territorio ejidal en las siguientes áreas: 1) Mulegé, 2) núcleo Bonfil y 3) Santa Martha (figura I). Cada área comprende una cantidad variada de rancherías y asentamientos humanos dispersos. 
Figura I

Área de estudio: a) Estado de Baja California Sur;

b) Municipio de Mulegé; c) Ejido Bonfil y las tres áreas en que se dividió para aplicar el TERP
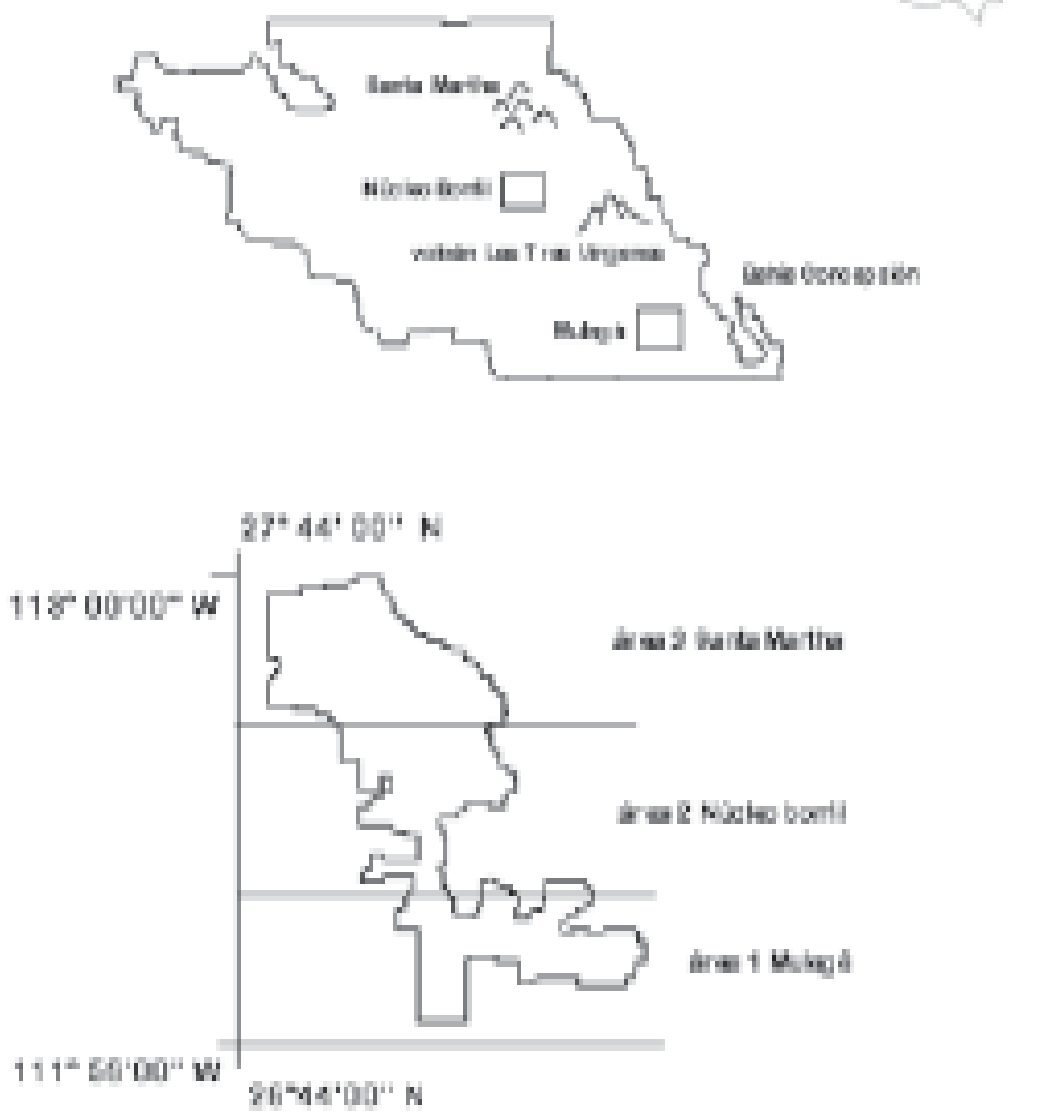

Fuente: Elaboración propia a partir del Registro Agrario Nacional (RAN), BCS, 2001. 


\section{Metodología}

El presente estudio es un trabajo sociológico con enfoque multidisciplinario (participaron investigadores sociales, economistas y biólogos) donde se utilizaron metodologías de investigación cualitativas, básicamente herramientas y técnicas usadas en la ERP. Además se hicieron observaciones directas en campo mediante una relación continua con las comunidades a lo largo de más de un año. Las fuentes primarias y documentales fueron los principales medios para obtener información. Se hicieron revisiones bibliográficas y cartográficas, entrevistas (con una guía semiestructurada), charlas informales, reuniones con grupos focales (líderes de las comunidades), mapas participativos (diagrama realizado por cada dueño de parcela donde incluyó las actividades y los objetos más importantes de su área de trabajo), análisis de calendario por estación y uso del tiempo diario por género, cronología de las comunidades (en reuniones convocadas, por medio de narraciones los habitantes estructuraron entre todos la historia de la comunidad), matrices de problemas prioritarios y sus soluciones (los que miembros de la comunidad consideraron más urgentes).

En cuanto a metodologías específicas por género, se aplicó: 1) análisis de calendario por estación para describir la distribución y ocupación de actividades y 2) uso del tiempo diario para hombres y mujeres en cada hogar, generalmente desarrollados por el padre y la madre (ambos anotaron sus actividades por cada temporada del año y por hora del día).

Previo a la aplicación del taller de ERP, miembros de la ONG Bonfil, Ambiente y Desarrollo, A.c. e integrantes del grupo de investigadores participantes mantuvieron una relación por espacio de un año con diversas personas de las comunidades del ejido, por medio de reuniones y pláticas informales. Durante estas comunicaciones se planteó la idea de evaluar el manejo de los recursos naturales. Cuando hubo consenso entre el grupo facilitador -la ONG, los investigadores y diversos miembros del ejidoen cuanto a que existía convicción e interés para aplicar la ERP, se emprendió el proceso de evaluación.

Con una duración de poco más de cuatro meses, el TERP se elaboró en tres etapas: a) introducción y sensibilización a la comunidad sobre los beneficios de la ERP, $b$ ) capacitación al grupo facilitador en el uso de las herramientas y técnicas, y c) aplicación del taller (tabla 1). 


\section{Tabla 1}

Etapas de aplicación de la ERP

\begin{tabular}{lll}
\hline \multicolumn{1}{c}{ Etapa } & \multicolumn{1}{c}{ Actividad } & Duración \\
\hline Primera etapa: & Revisión de información & Tres meses \\
Introducción y & bibliográfica especializada & \\
sensibilización a & sobre características & \\
las comunidades & ambientales de la región & \\
propuesta a las & Asambleas ejidales, reunión & \\
comunidades & de consejo y visitas a los & \\
para aplicar el TERP & hogares & Un mes \\
Segunda etapa: & Capacitación en las metodologías & \\
Capacitación del & y técnicas utilizadas en la ERP ONG: & \\
grupo promotor o & tres promotores & \\
facilitador & Grupo de investigadores: & \\
& dos promotores & Dos semanas \\
& Miembros de la comunidad: & \\
Tercera etapa: & Siete entrevistadores & \\
Aplicación del taller & Desarrollo del taller en las & \\
\hline Fununidades &
\end{tabular}

Fuente: Elaboración propia.

\section{Resultados}

\section{Descripción ambiental del ejido}

Este territorio comprende a la región geológica Sierra de la Giganta (Padilla y Troyo, 1991), cuya geodinámica incluye vulcanismo, sedimentación y mineralogía. De acuerdo con la clasificación fisiográfica, esta área pertenece a la provincia de Baja California, constituida por una región de sierras altas (400 a 600 msnm, excepto las serranías de Tres Vírgenes con altitudes máximas de 2,100 m) y bajas (lomeríos menores a $300 \mathrm{msnm}$ ). En cuanto al clima, se reconoce como zona muy seca o árida y seca cálida BW (h') s ('x)(I'), con temperatura media anual mayor a $22^{\circ} \mathrm{C}$, con un porcentaje de lluvia en verano o invierno de 5 a $10 \mathrm{~mm}$ y oscilación térmica extremosa (Salinas et al., 1991). En lo referente a la flora, comprende porciones de dos subdivisiones del desierto sonorense: desierto del Vizcaíno y una porción menor que es Costa del Golfo. Aproximadamente 9\% de la flora se reconoce como endémica de la región geográfica global y sus formas corresponden a árboles, arbustos, herbáceas, arrosetadas y cactáceas (columnares, cilíndricas, globosas y cespitosas) (León y Coria, 1992). Con respecto a la fauna, se considera que actual- 
mente los vertebrados de este lugar se conforman de una mezcla de la Región del Cabo y del macizo continental -sobre todo del sudoeste de Estados Unidos y del noroeste de México-, y se reconoce como fauna del Vizcaíno Sur, la cual se considera como una verdadera isla ecológica, pues está apartada del resto de la península por factores ambientales debido a que es uno de los desiertos más áridos de Norteamérica. Entre los ejemplares más representativos se encuentran: venado bura (Odocoileus hemionus peninsulae), borrego cimarrón (Ovis canadensis weemsi), zorra del desierto (Vulpes macrotis devia), puma (Felix concolor), gato montés (Linx rufus), coyote (Canis latrans), halcón peregrino (Falco peregrinus), águila real (Aquila chrysaetos), águila pescadora (Pandion haliaetus), entre otros (Galina et al., 1991, 2003).

\section{Características socioeconómicas}

Los asentamientos poblacionales en este ejido se dieron en diversas fechas. De acuerdo con los datos cronológicos obtenidos durante el TERP aplicado en el núcleo Bonfil, las primeras familias llegaron en 1977 provenientes del estado vecino de Baja California Norte, sobre todo del valle de San Quintín y Ensenada -aunque en general los orígenes de estas personas son los estados del centro y sur de México-, y sus principales actividades eran la agricultura y la ganadería. Poco tiempo después, en los inicios de 1980, se incorporaron al sistema ejidal de Bonfil los ranchos ubicados dentro de los límites oficiales del territorio ejidal, asentamientos localizados en la sierra de Santa Martha y Mulegé (figura I) cuyos pobladores en su mayoría pertenecen a familias nativas de la región descendientes directos de colonizadores extranjeros, principalmente europeos (Jordán, 1987). Así pues, el ejido Bonfil es una mezcla de costumbres, formas de pensar, necesidades y deseos diferentes, aspectos que con el tiempo les han dado características que los hacen parte de su entorno.

Actualmente la población se compone de 700 personas (155 ejidatarios con sus respectivas familias) influenciadas directamente con el sistema ejidal de Bonfil. Las comunidades están distribuidas de sur a norte (figura I) de la siguiente manera:

1) En Mulegé se encuentran 50 ejidatarios que se dedican a la agricultura (huertas de cítricos, dátil criollo y pastizales), la ganadería, la pesca y el turismo de playa (rentan predios en la franja costera de las playas de Santa Inés y 
Bahía Concepción). Alrededor de 25 ejidatarios más viven dispersos en poblados y rancherías de las serranías, desde Santa Águeda hasta el sur de San José de Magdalena, y sus principales actividades son la cría de ganado bovino y caprino, así como el cultivo de huertas frutales.

2) En la comunidad núcleo del ejido viven 50 ejidatarios dedicados al cultivo de una variedad mejorada de dátil y diversas hortalizas, legumbres y frutas (principalmente cítricos) y a la cría -para autoconsumo- de ganado bovino y caprino así como de aves de corral. Riegan los cultivos con agua que extraen de acuíferos; existen cinco pozos que funcionan por bombeo alimentados con energía eléctrica.

3) En San Francisquito y Santa Martha viven alrededor de 25 familias de ejidatarios que también crían ganado bovino y caprino, actividad que combinan con trabajos de curtiduría y manufactura de calzado típico de la región. El agua que utilizan tanto para el desarrollo de sus actividades como para el consumo humano, la toman de aguajes naturales.

En distintos grados, los ejidatarios hacen uso directo o indirecto de los recursos naturales que los circundan y todos coinciden en que estas prácticas han modificado los patrones poblacionales de algunas especies de fauna, por ejemplo, el venado ha sido diezmado pero la cantidad de liebres (Lepus californicus) se incrementó, otros presentan cambios constantes a corto plazo, como las víboras de cascabel (Crotalus sp) y el halcón peregrino (Falco peregrinus), así como algunos moluscos bivalvos cuyas poblaciones silvestres -según habitantes del ejido que se dedican a actividades pesqueras- aumentan y disminuyen entre ciclos que van de dos a 10 años.

En cuanto a las actividades cotidianas, tanto hombres como mujeres tienen una rutina bien definida durante el día. Generalmente las mujeres de Bonfil desarrollan las siguientes actividades: de 5:00 a 7:00 hrs. -se levantan una hora más temprano que los hombres- inician sus labores con la elaboración del desayuno y preparando a sus hijos para que asistan a la escuela; de 7:00 a 8:00 sirven el desayuno a quienes se quedan en casa y ponen los alimentos que el esposo lleva a su lugar de trabajo; entre las 8:00 y 13:00 hrs. limpian la casa, lavan ropa, cocinan los alimentos del medio día y reciben a los niños que regresan de la escue- 
la; de las 13:00 a las 16:00 continúan con la limpieza, riegan las plantas de su solar, reciben al esposo tras su jornada de trabajo en el campo, le ofrecen alimentos y comienzan a preparar la cena; de las 16:00 a las 19:00 ayudan a los hijos con las tareas y ordenan los útiles y ropas escolares para el día siguiente; de las 19:00 a las 21:00 sirven la cena, limpian los utensilios de cocina utilizados y generalmente se duermen una hora más tarde que los demás miembros de la familia. En cuanto a los cambios de rutina entre estaciones del año, ésta sólo se modifica durante las vacaciones marcadas por el calendario escolar.

Habitualmente, la jornada de los hombres que trabajan en actividades agropecuarias es como sigue: 6:00 a 8:00 hrs. se levantan, desayunan y salen de sus viviendas; entre las 8:00 y 11:00, inician algunas actividades como encender los motores del pozo de donde extraen el agua, comienzan el riego de sus parcelas y hacen diversas labores de labranza (dependiendo de la temporada, siembran, deshierban, riegan, cosechan etc.,); allí mismo toman un almuerzo entre las 11:00 y 12:00; continúan sus trabajos en el campo hasta las 15:00 o 16:00 hrs.; después regresan a sus casas a comer, descansan un rato y aproximadamente a las 20:00 o 21:00 hrs. cena toda la familia.

\section{Recursos naturales: usos en tiempo, problemas y soluciones priorizados por la comunidad}

Después de realizar el TERP encontramos que en el núcleo Bonfil (tabla 2) la principal actividad en la comunidad es el manejo de fauna silvestre a través de una unidad de manejo ambiental (UMA) que incluye la actividad cinegética, la agricultura, la ganadería y la caza para autoconsumo. Los principales problemas, en orden de importancia según los habitantes del ejido, son: altos costos de la energía eléctrica, carencia de créditos, suelos pobres, escasez de agua, falta de alimento para el ganado, ausencia de información sobre el manejo de la UMA y poca disponibilidad de leña cerca del poblado. Asimismo, algunas soluciones que propusieron son: acordar un subsidio con la Comisión Federal de Electricidad (CFE) y agrupar un mayor número de ejidatarios (para pagar menos); asociarse con grupos financieros; implementar programas de labranza de conservación y de compostas y abonos; solicitar apoyo del gobierno para construir represas; crear una asociación entre ganaderos y agricultores para ofrecer información acerca del Programa uma Borrego Cimarrón a la comuni- 


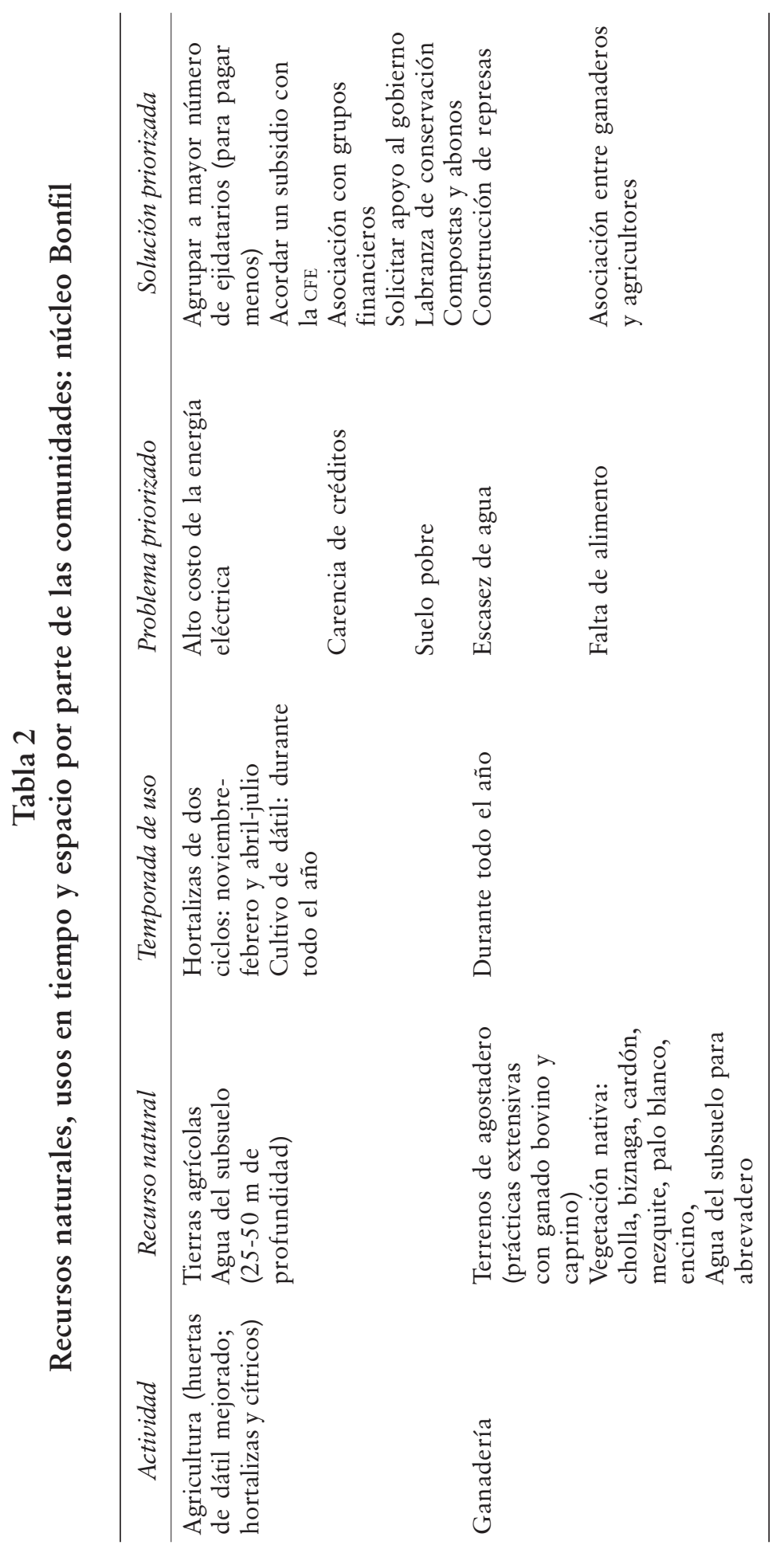


Economía, Sociedad y Territorio, vol. viII, núm. 26, 2008, 451-476

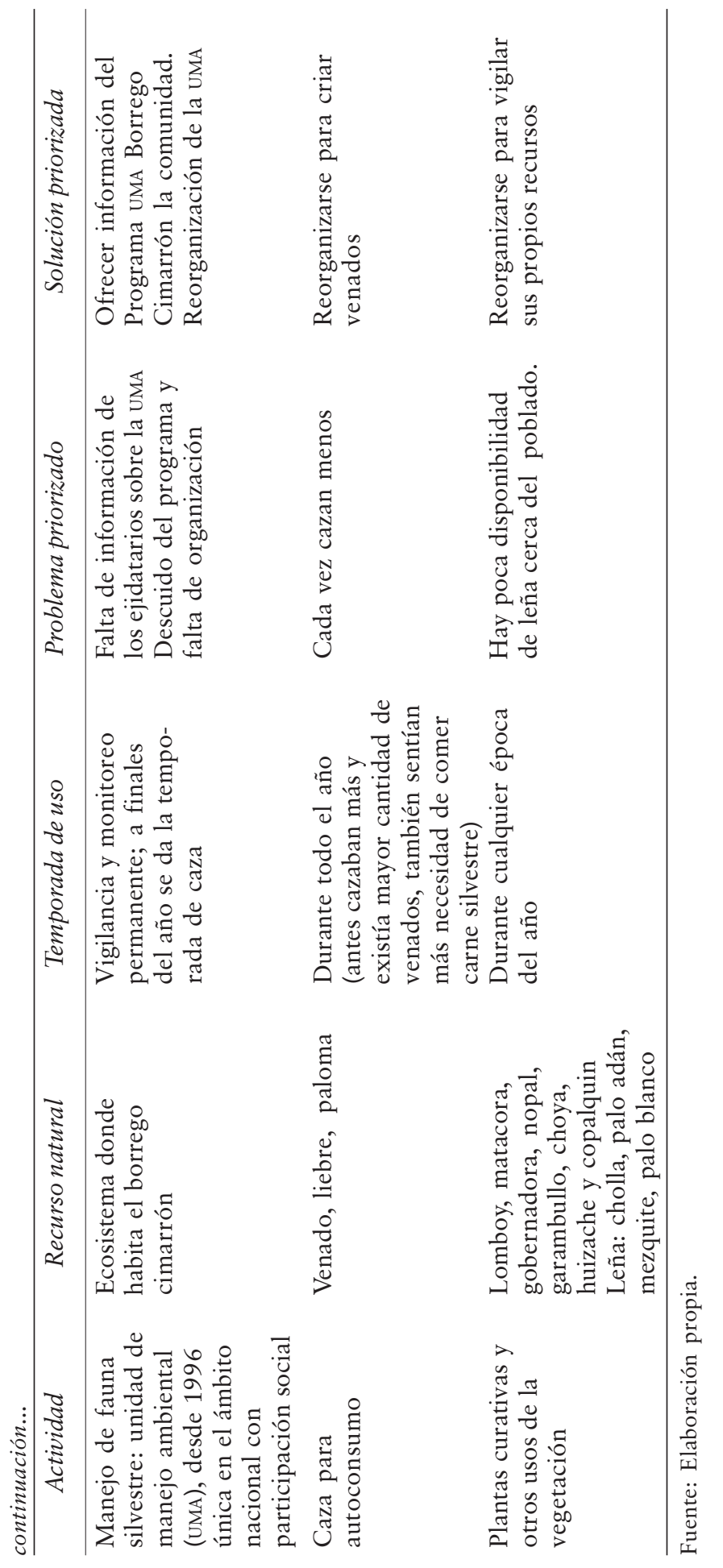


dad; reorganizar la UMA y coordinarse para vigilar sus propios recursos. Quienes elaboramos este estudio coincidimos con la identificación de la problemática, así como con las soluciones propuestas.

En el núcleo Santa Martha (tabla 3) las principales actividades son el ecoturismo (pinturas rupestres), la ganadería, la caza para autoconsumo y la agricultura en baja escala. Los problemas más apremiantes son: disminución del ganado caprino debido a la plaga del gusano y al ataque de pumas; poca organización del Instituto Nacional de Antropología e Historia (INAH) y falta de información acerca de las pinturas rupestres; insuficiencia de leña cercana al poblado y menores posibilidades de caza. Algunas soluciones propuestas por ellos mismos son: integrar una UMA para el manejo de fauna silvestre que ataca al ganado; crear un convenio con el gobierno del estado para constituir un fondo o seguro que cubra la pérdida de ganado; implementar una buena promoción sobre la vacuna para atacar la plaga del gusano; gestionar ante el INAH, en el ámbito estatal, el fortalecimiento, capacitación y mejoramiento de la calidad de vida de los guías de Santa Martha para la visita a las pinturas rupestres; hacer promoción local, nacional e internacional y reorganizarse para vigilar sus propios recursos.

En el núcleo de Mulegé (tabla 4) las principales actividades son la ganadería, la agricultura, la pesca, el turismo y la caza para autoconsumo. Los problemas más apremiantes que se encontraron fueron falta de agua; fuertes costos de electricidad; nula organización y falta de equipo en el sector pesquero; impuestos altos para iniciar un negocio; menores posibilidades de caza; y poca leña disponible cerca del poblado. Las soluciones que propusieron son: desasolvar los aguajes; conseguir equipo para traer agua hacia los ranchos; buscar acuerdos y subsidios de la CFE; organizar cooperativas y buscar financiamiento para equipo de pesca; negociar con las autoridades pertinentes impuestos menos altos; y reorganizarse para vigilar sus propios recursos.

\section{Conclusión}

El uso de herramientas participativas en actividades de desarrollo se debe principalmente a que se ha reconocido, a través de diversas experiencias mundiales, que el proceso de desarrollo no es lineal ni sencillo, donde de la situación A se pasa a la $\mathrm{B}$, en línea recta. Más bien, el desarrollo es un estado dinámico en el 
Economía, Sociedad y Territorio, vol. vIII, núm. 26, 2008, 451-476

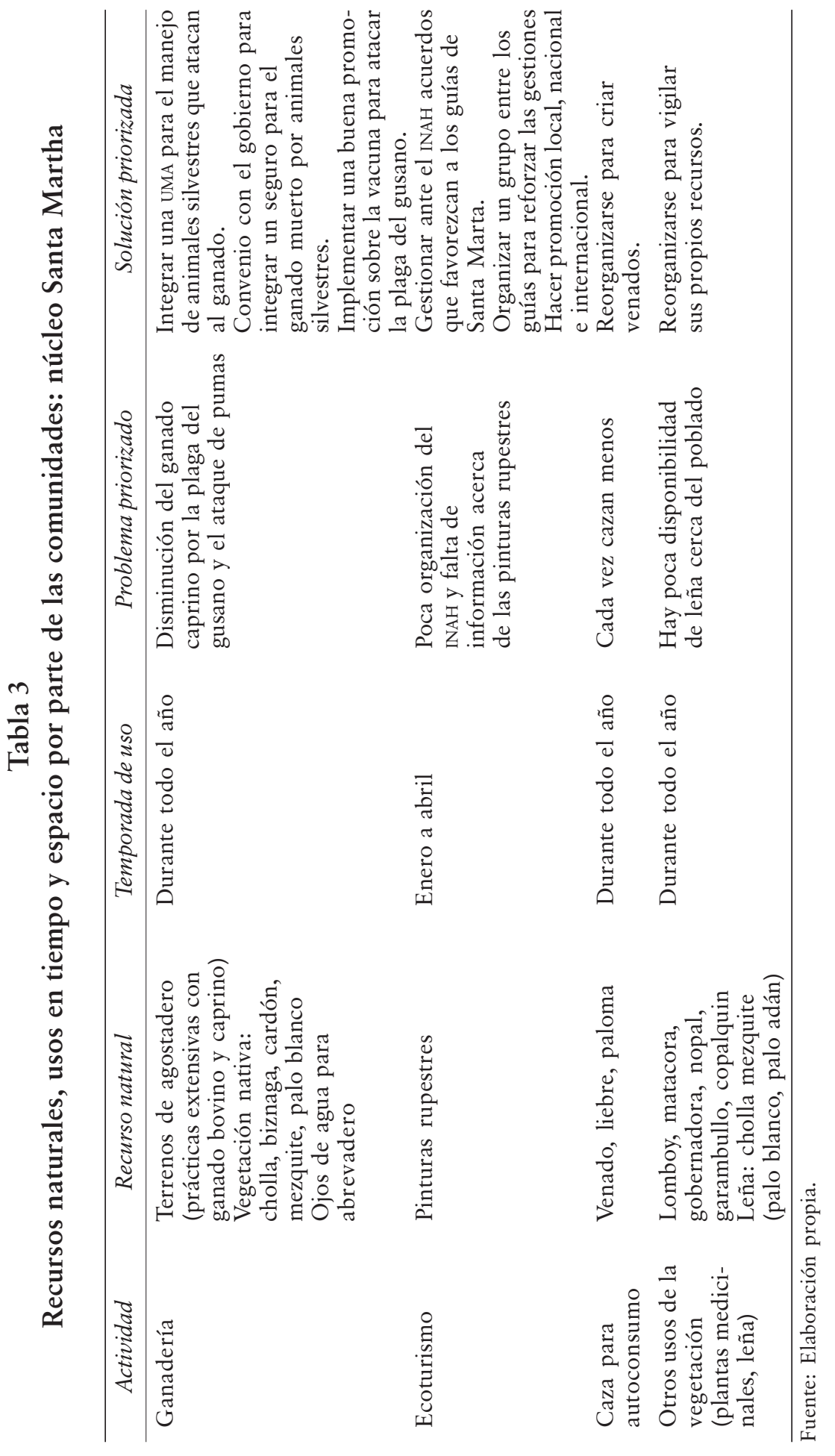




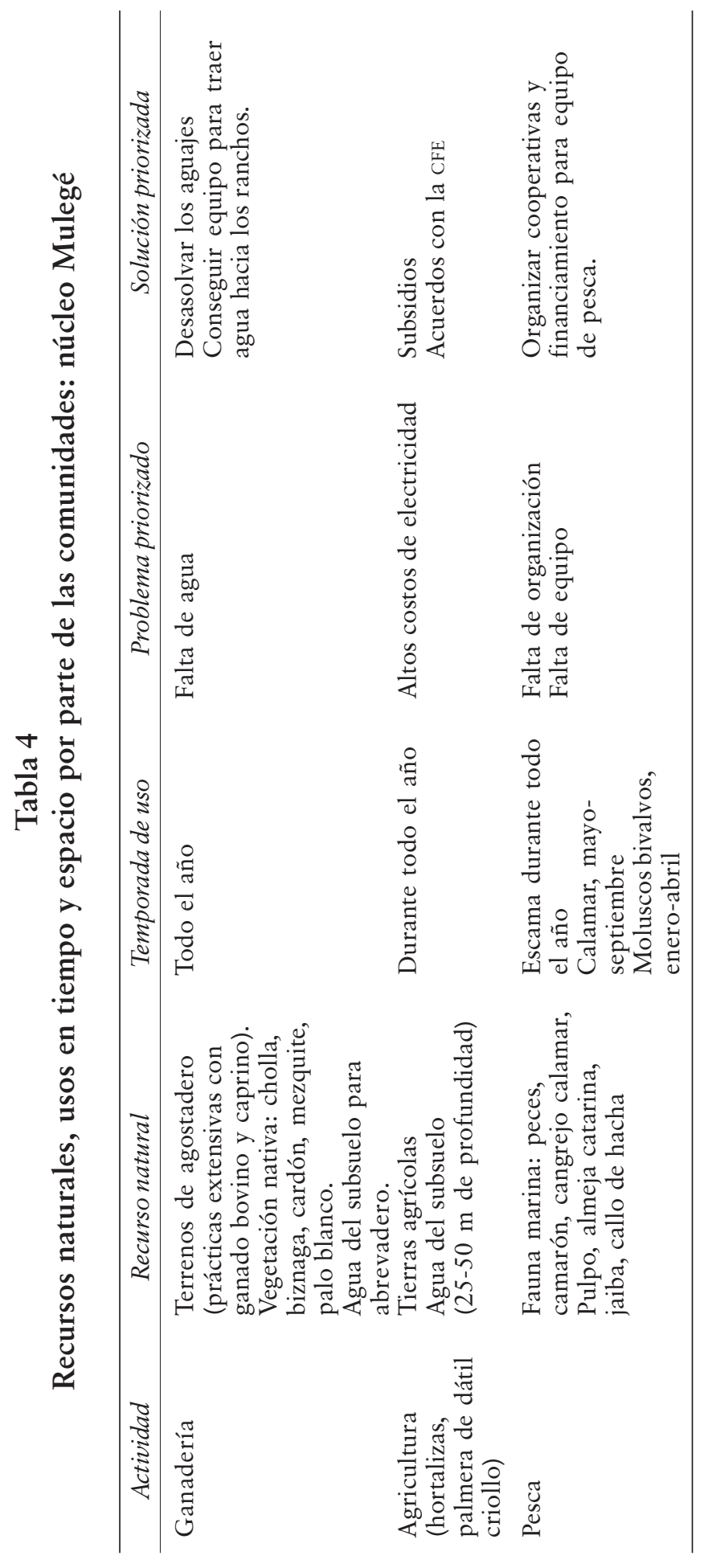


Economía, Sociedad y Territorio, vol. vIII, núm. 26, 2008, 451-476

469

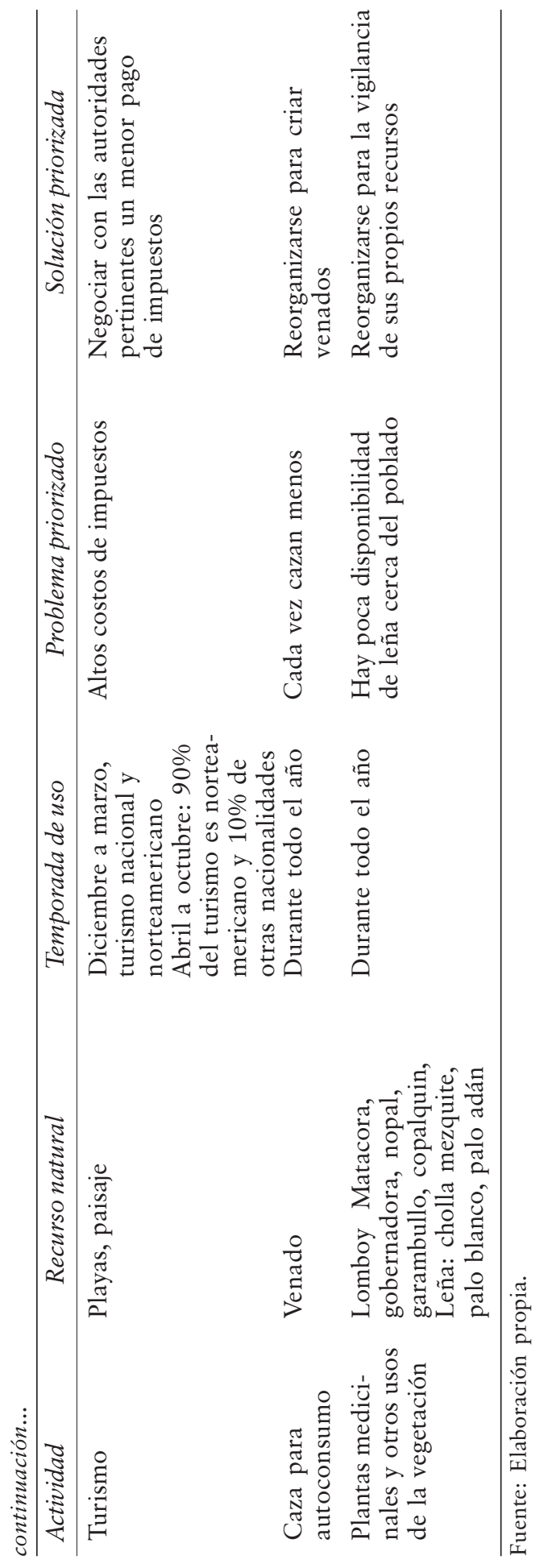


que la participación hace posible pasar gradualmente de una pasividad casi completa (ser beneficiarios) al control de su propio proceso (ser actor del autodesarrollo) (Geilfus, 2001).

Así, con la aplicación del terp en el ejido Bonfil, las personas dedicadas a la actividad agropecuaria se dieron cuenta de lo poco organizadas que estaban; mientras los ganaderos carecían de alimentos y agua para sus animales, los agricultores podrían proporcionarlos mediante un poco de iniciativa y coordinación entre ellos, y de esta manera ambos grupos se verían beneficiados.

Tomaron conciencia de cómo cada comunidad llevaba a cabo diferentes actividades acordes con su entorno natural: los habitantes del núcleo Bonfil dedicados a la agricultura, la ganadería y el manejo de fauna silvestre; los rancheros de Santa Martha, ganadería extensiva y ecoturismo (pinturas rupestres cercanas); y los pobladores de la zona de Mulegé, agricultura, ganadería, pesca y turismo de playa.

Con el TERP, en el ejido Bonfil se elaboró un documento conocido como Plan de Manejo Comunitario de los recursos naturales, el cual es esencial para un mejor aprovechamiento de estos recursos. El trabajo desarrollado muestra las bondades de la labor participativa: la combinación del conocimiento académico con el tradicional para generar opciones que concilien el uso de los recursos y su conservación (Bocco et al., 2000).

Realizar este tipo de investigación participativa acerca del verdadero uso de los recursos naturales y su importancia para una comunidad rural nos acerca más a la realidad. Esta metodología es importante para implementar estrategias prácticas para el buen uso y conservación de los recursos, al tiempo que brinda la oportunidad para elaborar proyectos de desarrollo viables para las comunidades involucradas.

Si bien la estructura científica aún no incorpora claramente la relevancia de este tipo de investigación en su sistema, conseguir resultados concretos y verificar enfoques en la realidad $-y$ no solamente en juicios de pares-, parece una perspectiva atractiva para grupos de universitarios interesados en frenar el deterioro de los recursos naturales (Bocco et al., 2000).

\section{Agradecimientos}

Queremos agradecer y hacer un reconocimiento especial a la asociación Bonfil, Ambiente y Desarrollo, A.C. Asimismo, reconocemos la colaboración en la aplicación del TERP de las siguien- 
tes personas: a las biólogas Silvia Uscanga A. y Genny Canúl, a los ingenieros Rolando A. Alonzo y Elvia Verónica García Chávez, a los estudiantes Rosa García Chávez y Luis Chavarría Romero. A la mesa directiva del ejido Bonfil, al señor Armando Naranjo R. y, también de manera muy especial, a todos los miembros de las comunidades de Bonfil, así como a dos revisores que contribuyeron para mejorar este trabajo.

\section{Bibliografía}

Anta-Fonseca Salvador (2006), "Conservación de la biodiversidad en tierras indígenas: una propuesta surgida de las comunidades rurales", La Jornada Ecológica, 4 de diciembre, México, http:/www.unam.mx/2006/12/04/ecof.html, 12 de diciembre de 2006.

Berkes Fikret, Robin Mahon, Patrick McConney, Richard Pollnac y Robert Pomeroy (2001), Managing small-scale fisheries alternative, directions and methods, International Development Research Center, Ottawa, 285 pp.

Bocco, Gerardo, Alejandro Velázquez y Alejandro Torres (2000), "Ciencia, comunidades indígenas y manejo de recursos naturales. Un caso de investigación participativa en México", Interciencia, 25(2), Caracas, pp. 64-70.

Carabias, Julia, Enrique Provencio y Carlos Toledo (1994), Manejo de recursos naturales y pobreza rural, Universidad Nacional Autónoma de México-Comisión Federal de Electricidad, México, 137 pp.

Centro Internacional para el Ambiente y el Desarrollo-Instituto de los Recursos Mundiales (1993), El proceso de evaluación rural participativa, Centro Internacional para el Ambiente y el Desarrollo-Instituto de los Recursos Mundiales, Montevideo, 103 pp.

Comisión Nacional de Áreas Naturales Protegidas (CONANP) (1999), URL, <http://www.ine.gob.mx/ucanp/listaanpesta.php3 >.

Chambers, Roberts (1983), Rural development: Putting the last first, Longmans, Londres, pp.150. 
Chambers, Roberts (1991), “Farmer's practices, professionals and participation: Challenges for soil and water management", documento presentado en Farmer's Practices and Soil and Water Conservation Programes, ICRISAT, India.

Chambers, Roberts (1992a), "The self-deceiving state: Psychosis and therapy", IDs Bulletin, 23(4), Institute of Development Studies. Reino Unido, pp. 31-42.

Chambers, Roberts (1992b), "Methods for analysis by farmers: The professional challenge", documento presentado en el 12th Annual Symposium of the Association for FSR/E, East Lansing, Michigan State University, Estados Unidos.

Chambers, Roberts (1993), Challenging the professions: Frontiers for rural development, Intermediate Technology Publications, Londres.

Chambers, Roberts (1994), The origins ans practice of Participatory Rural Appraisal, World Development Vol. 22, No. 7. pp. 953-969.

Chambers, Roberts (1995), "Rural appraisal: Rapid, relaxed and participatory”, IDS Discussion Paper 311, Brighton, IDS.

Chambers Roberts (1996), "Evaluación rural participativa", en <http: www.neareast.org/main/lrc/biblio/researchandevaluation. pdf>, 21 de marzo de 2001.

Galina-Tessaro, Patricia, Aradit Castellanos-Vera, Enrique Troyo-Diéguez, Gustavo Arnaud F. y Alfredo Ortega (2003), "Lizard assemblages in the Vizcaino Biosphere Reserve, Mexico", Biodiversity and Conservation, 12, Springer Netherlands, pp. 1321-1334.

Galina, Patricia, Sergio Álvarez S., R. González y Sonia Gallina (1991), "Aspectos generales sobre la fauna de vertebrados", en Alfredo Ortega y Laura Arriaga (eds.), La reserva de la bios-fera El Vizcaino en la península de Baja California, México, Cibnor, s.C., 242 pp.

Geertz, Clifford (1994), Conocimiento local, Barcelona, Paidós. 
Geilfus, Frans (2001), 80 herramientas para el desarrollo rural participativo: diagnóstico, planificación, monitoreo y evaluación, IICA-Sagarpa, México, 208 pp.

González-García, Carlos (2001), "Nueva agresión contra la comunidad indígena", La Jornada, 1 de mayo, Ojarasca, México, 49, URL, <http://www.jornada.unam.mx/2001/05/01/ oja-portada.html $>$.

Granich, Iisley Catarina (2006), "Diez años de trabajo regional en la zona centro de Guerrero", La Jornada Ecológica, 4 de diciembre, México, http:/www.jornada.unam.mx/ 2006/12/04eco-f.html, 10 de diciembre de 2006.

INEGI (Instituto Nacional de Estadística, Geografía e Informática) (2000a), XII Censo de población y vivienda, México, INEGI.

INEGI (2000b), Catálogo interinstitucional de núcleos agrarios, México, INEGI.

Jordán, Fernando (1987), El otro México. Biografía de Baja California. Frontera, Secretaría de Educación Pública, México, $303 \mathrm{p}$.

Leewis, Cees (2000), “Reconceptualizing participation for sustainable rural development: towards a negotiation approach", Development and Change, 31, Westview Press, co, pp. 931-959.

León de la Luz, J. Luis y Rocío Coria (1992), Flora icnográfica de Baja California Sur, Cibnor, s.C., México, 156 pp.

Macías-Cuellar, Humberto, Oswaldo Valdez-Téllez, Patricia Dávila-Aranda y Alejandro Casas-Fernández (2006), "Los estudios de sustentabilidad”, Ciencias 81, Universidad Nacional Autónoma de México, México, pp. 20-31.

Martínez-Alvarado, Oscar, Venancio Cuevas-Reyes, Alfredo Loaiza-Meza, Tomas Moreno-Gallegos, J. Esteban ReyesJiménez,Oscar Palacio-Velarde y José Luís Guzmán-Rodríguez (1999), Desarrollo sostenible del sistema agrosilvopastoril en el sur de Sinaloa. Red de Gestión de 
Recursos Ambientales. Fundación Rockeffeller. México, 94 pp.

Negrete Gerardo y Gerardo Bocco (2003), “El ordenamiento ecológico comunitario: una alternativa de planeación y participación en el contexto de la política ambiental de México", Gaceta Ecológica, 68, Instituto Nacional de Ecología, México, pp. 9-22.

Padilla, Pedrín y Enrique Troyo (1991), “Geología”, en Alfredo Ortega y Laura Arriaga (eds.), La reserva de la biosfera El Vizcaino en la península de Baja California, Cibnor, s.c., México, $242 \mathrm{pp}$.

Pool-Novelo, Luciano, Noe Martínez-León, Manuel Diaz-Ruiz, Antonio López-Meza, Rosa Zúñiga-López, Oscar Sánchez-Carrillo, José Pérez, Rosa Pérez, Reyna Moguer-Viveros y Manuel Roberto Parra-Vazquez (1997), "El ordenamiento territorial en Santa Martha, municipio de Chenalhó, Chiapas", Ecosur, URL, en <http://www.laneta. apc.org/rock/ecosur03.htm>, 15 de mayo de 2006.

Pretty, Jules y Richard Sandbrook (1991), “Operationalizing sustainable development at the community level: Primary environmental care", documento presentado en la DAC Working Party on Development Assistance and the Environment, París, OECD.

Pretty, Jules y Roberts Chambers (1993), “Towards a learning paradigm: New professionalism and institutions for sustainable agriculture, IDs Discussion Paper 334, Brighton, IDS.

Pretty, Jules, Irene Guijt, Scoones y John Thompson (1995), A trainer's guide to participatory learning and action, IIED Training Materials Series 1, Londres, IIED.

Salinas-Zavala, Cesar, Rocio Coria-Benet y Eva Díaz-Rivera (1991), "Climatogía y meteorología", en Alfredo Ortega y Laura Arriaga (eds.), La reserva de la biosfera El Vizcaíno en la peninsula de Baja California, México, Cibnor, s. C.

Valarezo-Ramon, Galo (1995), "Metodologías participativas: el estado de la cuestión en América Latina”, Bosques, Árbo- 
les y Comunidades Rurales, 25, <http://wwwfao.org/docrep/x0221s/x0221s00.htm\#topofpage, consultada el 10 de diciembre de 2006>.

Vallejo-Nieto, Mirna Isela, Clara Balderrama-Barbeitia, Elia Margarita Chablé-Can, Dolores O. Molina-Rosales, Griselda Escalona-Segura, s/f. Diseño e implementación del sistema de monitoreo y evaluación participativa: la experiencia en Calakmul, Campeche, Ecosur Unidad-Campeche.

Vázquez Cruz y Miriam Montenegro-Penagos (1999), Manual de herramientas para la intervención en comunidades pesqueras y acuícolas, MADR-INPA, Santa Fe de Bogotá, 177 pp.

Recibido: 7 de febrero de 2006. Reenviado: 25 de mayo de 2006. Aceptado: 29 de enero de 2007.

Magdalena Lagunas Vázquez. Es candidata a doctora en ciencias, en el posgrado de Ciencias Marinas y Costeras (Cimaco), por la Universidad Autónoma de Baja California Sur, con especialidad en manejo sustentable de la zona costera. Es autora o coautora de cinco artículos de investigación original y de un capítulo de libro. Ha dirigido y participado en ocho proyectos de investigación y en 18 proyectos de restauración ecológica e impacto ambiental, entre los que destaca la coordinación del ordenamiento ecológico en acuacultura para Bahía Magdalena, BCS, México.

Luis Felipe Beltrán Morales. Es licenciado en economía por la Universidad Autónoma de Baja California Sur (UABCS); doctor en ciencias ambientales por el Centro eula de la Universidad de Concepción, Chile; investigador del Centro de Investigaciones Biológicas del Noroeste (Cibnor, s.c.); miembro del Sistema Nacional de Investigadores (SNI) y de la Asociación Latinoamericana de Economistas Ambientales; es profesor de la maestría en economía del medio ambiente y recursos naturales de la UABCS. Es autor o coautor de 27 artículos de investigación original; editor de dos libros y autor de ocho capítulos de libros. Ha participado en 38 estudios de impacto ambiental y dirigido alrededor de 20. Actualmente es coordinador de estudios ambientales del Cibnor. 
José Urciaga García. Es doctor en ciencias económicas y empresariales por la Universidad Autónoma de Barcelona, España; Miembro del Sistema Nacional de Investigadores, nivel II. Profesor-investigador titular del Departamento de Economía de la Universidad Autónoma de Baja California Sur. Profesor de la Maestría en Economía del Medio Ambiente y Recursos Naturales (UABCS) y del posgrado (especialidad, maestría y doctorado) en ciencias marinas y costeras, UABCS. Autor o coautor de 4 libros, 10 capítulos de libro, 27 artículos de investigación y ha participado en el desarrollo de 14 proyectos de investigación en el área de desarrollo. Actualmente es director de investigación interdisciplinaria y posgrado de la Universidad Autónoma de Baja California Sur (UABCS) .

Alfredo Ortega Rubio. Es doctor en ciencias con especialidad en ecología por el Instituto Politécnico Nacional (IPN); premio nacional en Reconocimiento a la Conservación de la Naturaleza 2003, Categoría Académica y de Investigación; premio al Mérito Nacional Forestal y de la Vida Silvestre 1993; miembro del Sistema Nacional de Investigadores (SNI), nivel III. Es autor o coautor de 125 artículos de investigación original; editor de 13 libros y autor de 45 capítulos de libros. Ha dirigido 34 proyectos de manejo de recursos, algunos de ellos permitieron crear y desarrollar las reservas de la biosfera de Sierra de la Laguna, Desierto del Vizcaíno, e Islas Revillagigedo. Asimismo, ha dirigido 78 proyectos de restauración ecológica e impacto ambiental. 